\title{
Comparison between Posterior Polymorphous Dystrophy and Congenital Hereditary Endothelial Dystrophy of the Cornea
}

\author{
A. C. E. McCARTNEY and C. M. KIRKNESS \\ London
}

\begin{abstract}
Summary
Corneal discs from 10 cases of posterior polymorphous dystrophy (PPD) and 20 cases of congenital hereditary endothelial dystrophy (CHED) were compared and contrasted using light and electron microscopy. Secondary epithelial changes were similar in both diseases but spheroidal degeneration of stroma was seen more commonly in dominant CHED and not at all in PPD, when band, calcific, keratopathy was commoner.

Changes at the level of Descemet's membrane showing failure to regulate growth were seen in recessive CHED whereas dominant CHED and PPD were both associated with development of a fibrillary posterior collagen layer (PCL). Grotesquely banded PCL was also seen in some cases of PPD. Endothelial changes included vacuolation, development of microvilli and desmosomes in both diseases but multilayering was more common in PPD. The viscous layer of the cornea was seen by TEM in one case of PPD.
\end{abstract}

Posterior polymorphous dystrophy (PPD) and congenital hereditary endothelial dystrophy (CHED) have been considered by some authors to be similar diseases, ${ }^{1,2}$ possibly due to abnormal final differentiation of neural crest cells. ${ }^{3}$ Most patients with PPD have bilateral non-progressive, asymptomatic disease, which rarely requires keratoplasty, hence the small numbers of histological reports in the literature compared to the relatively high incidence of the disease in the population. CHED, in contrast, is a much less common disease, especially in the autosomal dominant form, but a much greater proportion of patients require surgery and their discs are available for histology. ${ }^{4}$ The largest published series of PPD is that of Krachmer ${ }^{5}$ who reports on the clinical and pathological findings in 13 patients. The next largest ${ }^{6}$ is of nine patients. Our series differs from the former ${ }^{5}$ since none of our 10 cases has glaucoma, ${ }^{5,7}$ nor do they have features of Chandler's syndrome,${ }^{8}$ broad iris synechiae,,${ }^{9,10}$ or anterior chamber dysgenesis. One of our cases did however have Alport's syndrome with associated deafness and hereditary nephritis. ${ }^{12}$

\section{Material and Methods}

Since this paper contrasts the findings in 10 cases of PPD with those of 20 cases of CHED reported separately, ${ }^{4}$ only the PPD patients are described here. Five of the ten patients had attended Moorfields Eye Hospital and five other corneas were submitted to the Institute for analysis. All patients underwent penetrating keratoplasty due to progressive disease or decompensation following cataract surgery and had been observed clinically for several years. Slit-lamp examination showed vesicles, plaques or 'snail tracks' at the level of 
the endothelium. Descemet's membrane was often thickened and band keratopathy was common. All 10 patients required keratoplasty for increasing corneal oedema, with increased thickness on pachymetry.

\section{Results}

The mean age at keratoplasty was 51 years in contrast to the patients with CHED, where the recessive group underwent surgery at 10.5 years and the dominants, who tended to present later and were initially less eager to undergo surgery, were operated on at a mean age of 34.4 years.

The epithelial changes, usually regarded as secondary, included thinning and oedema in both diseases but subepithelial fibrosis was commoner in PPD (Table I). Stromal spheroidal degeneration was noted in autosomal dominant CHED but not in PPD, whereas band calcific keratopathy, as had been clinically demonstrated, was commoner in PPD. Vascularisation was not a feature of PPD. The thickness of Descemet's membrane was measured by graticule at $\times 400$ using light microscopy and the results are shown in
Figure 2. The patients with PPD showed broad but nodular thickening whereas in CHED the thickness of Descemet's membrane (DM) was more uniform. The mean thickness of DM in autosomal dominant patients was $13.4 \mu \mathrm{m}$ and in recessives $14.5 \mu \mathrm{m}$ whereas the maximum thickness of DM in PPD was much greater when compared to the predicted thickness, ${ }^{13}$ with a mean of $21.5 \mu \mathrm{m}$ (Fig. 1).

Electron microscopy showed much greater contrasts between the two diseases. In CHED the thickening in recessive disease is associated in some cases with unrestricted growth of fetal type of Descemet's membrane, causing great thickening of the anterior banded zone (ABZ). In some cases, posterior non-banded zone (PNBZ), with basement membrane and occasional foci of long spaced collagen, was also thickened. ${ }^{4}$ Some cases of recessive disease but all cases of dominant disease showed a posterior collagenous layer of the fibrillary or birds nest pattern ${ }^{4}$ and it was this type of more non-specific thickening of Descemet's membrane by a fibrillary PCL, ${ }^{14}$ that was observed in 62.5 per cent of the

Table I Comparison between PPD and CHED

\begin{tabular}{|c|c|c|c|}
\hline & \multirow[b]{2}{*}{$P P D^{10}$} & \multicolumn{2}{|c|}{$C H E D^{20}$} \\
\hline & & $A D^{6}$ & $A R^{14}$ \\
\hline \multicolumn{4}{|l|}{ Epithelium } \\
\hline Thinning & $50 \%$ & $80 \%$ & $31 \%$ \\
\hline Oedema & $40 \%$ & $50 \%$ & $81 \%$ \\
\hline \multicolumn{4}{|l|}{ Bowman's zone } \\
\hline Subepithelial fibrosis & $90 \%$ & $60 \%$ & $19 \%$ \\
\hline Calcification & $50 \%$ & $20 \%$ & 0 \\
\hline \multicolumn{4}{|l|}{ Stroma } \\
\hline Vascularisation & $10 \%$ & $30 \%$ & 0 \\
\hline Spheroidal degeneration & 0 & $50 \%$ & 6 \\
\hline Descemet's membrane & Maximum (nodular) & (Diffuse) & (Diffuse) \\
\hline Increased thickness & $\overline{\mathrm{x}} 21.5 \mu \mathrm{m}$ & $\overline{\mathrm{x}} 13.4 \mu \mathrm{m}$ & $\overline{\mathrm{x}} 14.5 \mu \mathrm{m}$ \\
\hline \multicolumn{4}{|l|}{ E.M. of Descemet's } \\
\hline $\mathrm{ABZ}$ & Normal & Normal & Thick $20 \%$ \\
\hline PNBZ & Thin & Thin & Thick \\
\hline PCL fibrillar & $62.5 \%$ & $100 \%$ & $16 \%$ \\
\hline Caricature banded & $37.5 \%$ & 0 & 0 \\
\hline Endothelium pigmentation & $10 \%$ & $40 \%$ & $56 \%$ \\
\hline Multilayering & $60 \%$ & $25 \%$ & $20 \%$ \\
\hline Many microvilli & $62.5 \%$ & 0 & $30 \%$ \\
\hline Vacuolation & $62.5 \%$ & $25 \%$ & $83 \%$ \\
\hline Desmosomes & $37.5 \%$ & 0 & $10 \%$ \\
\hline
\end{tabular}



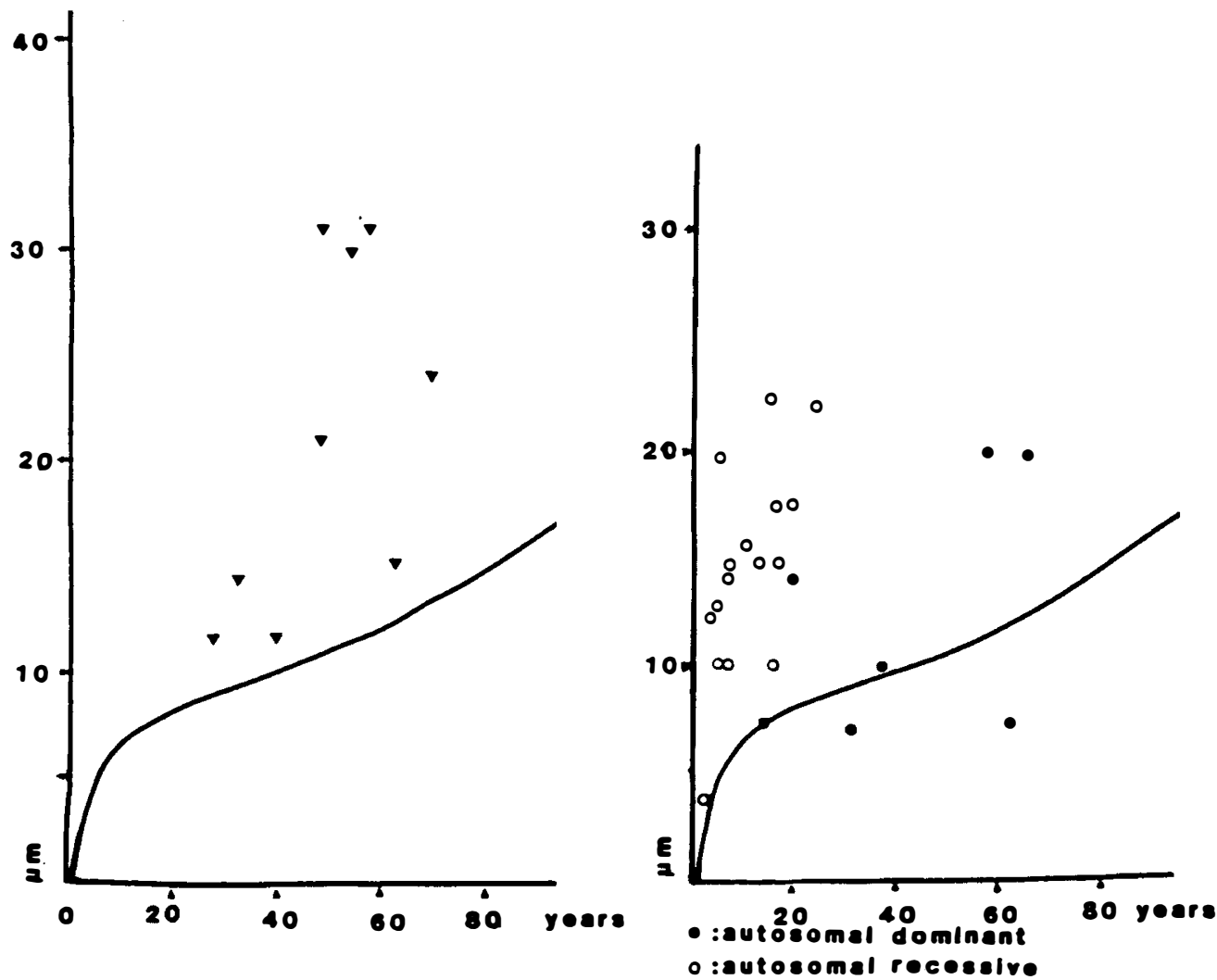

Fig. 1(a) Maximum thickness of DM in PPD against age (curve is predicted value for age).

(b) Mean thickness of DM in CHED (curve is predicted value for age).

patients with PPD. The rest of the patients showed a grotesque caricature of $\mathrm{ABZ}$ banded material in a banded PCL, ${ }^{14}$ where stacks or sheets of long (or wide) spaced collagen mixed between basement membrane and odd strands of collagen mimicked the orderly arrangement of ABZ. Most of the cases of PPD showed thinning of the normal posterior non-banded zone but the anterior banded zone was normal.

Endothelium showed less evidence of pigmentation with melanin in PPD, only being seen in one case, whereas 40 per cent of autosomal dominant and 56 per cent of recessive cases had endothelial cells with melanin within them but conversely multilayering visible with light microscopy was much commoner in PPD where 60 per cent of the cases showed it, compared to 20 per cent in autosomal recessive disease and 25 per cent in autosomal dominant cases.
Using both scanning (SEM) and transmission electron microscopy (TEM) the multilayering of cells was more dramatic, in PPD multilayering was seen both in association with nodular thickening of the DM and in areas where more normal thickness could be seen. Multilayering of cells (Fig. 2) was often less apparent in SEM but often similar cells, bristling wtih microvilli, could be seen beneath a peeling top layer of cells. Microvilli, often in hundreds, could be seen over the surface of cells in PPD especially, where 62.5 per cent of cases had them, compared to only 30 per cent of autosomal recessive CHEDs and no case of autosomal disease (Figs. 2 and 3 ).

Vacuolation of the endothelial cell layer, seen at light microscopy, was confirmed by transmission electron microscopy. The vacuoles are intercellular cisterns ${ }^{15}$ often seen beside effete cells, in conjunction with multi- 


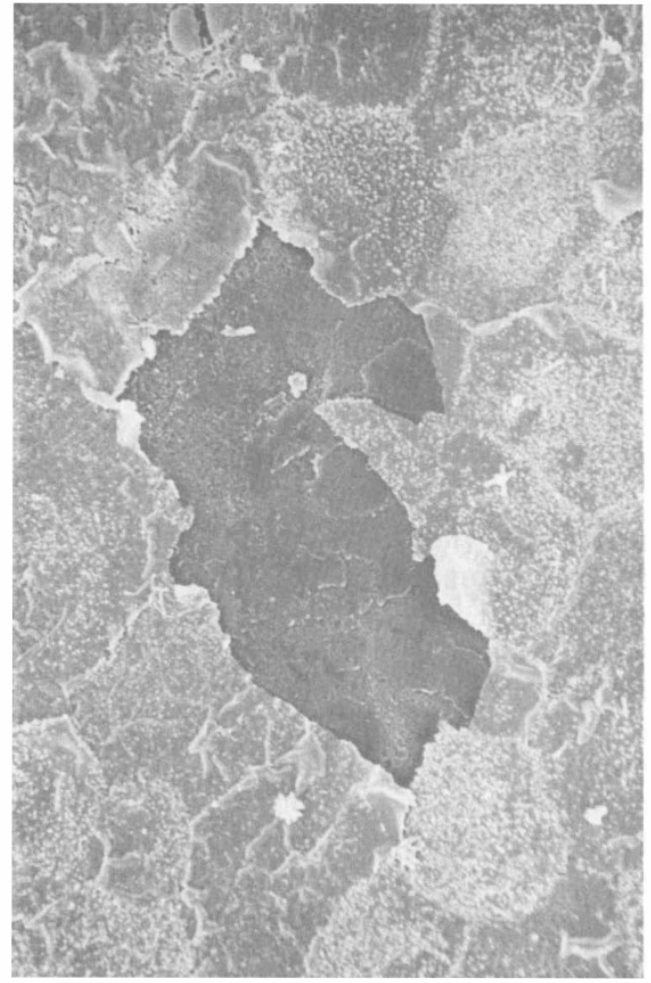

Fig. 2 Microvilli seen on top surface and on peeled under-surface of multiple layered endothelial cells in PPD. $(S E M \times 600)$

layering by more viable cells. Development of hemidesmosomes adjacent to Decemet's membrane or posterior collagenous layer, was seen in cases of multilayered PPD and was noted in conjunction with development of desmosomes in between the multilayered cells. This was seen in 40 per cent of cases of PPD, especially when layers of cells exceeded 2 cells thick (Fig. 4). In a case of CHED previously noted as unusual, ${ }^{4}$ similar multilayering with development of more strongly marked zonulae occludens was seen but true desmosomes were only seen between cells in the uppermost layers. Thick viscous layer was seen in one case of PPD.

\section{Discussion}

Posterior polymorphous dystrophy and congenital hereditary endothelial dystrophy have been stated to be similar diseases, occurring as part of the spectrum of a single entity based on

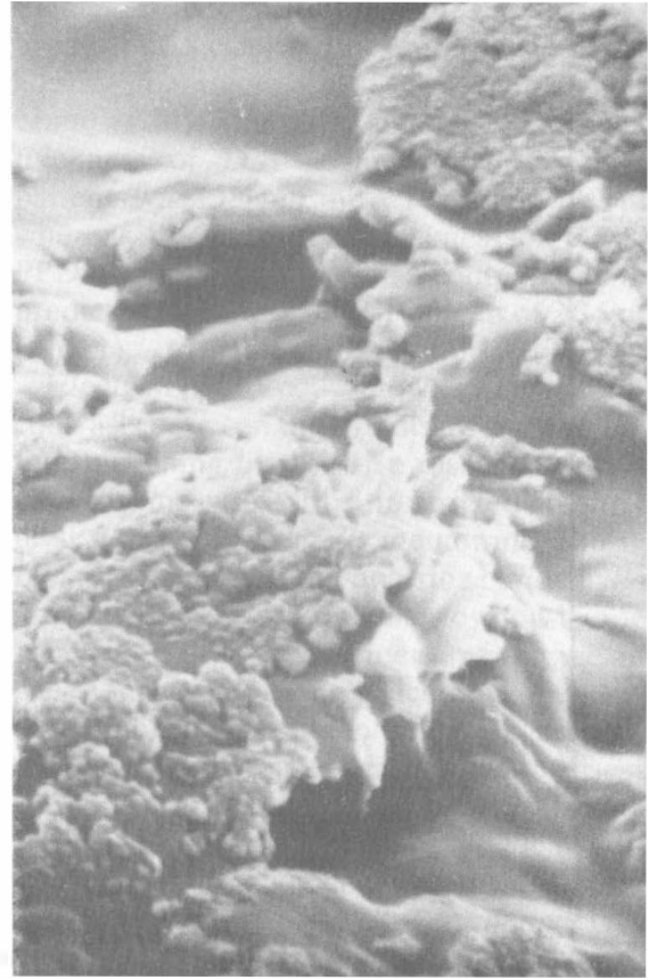

Fig. 3 Protruding microvilli on surface of 'epithelialised' endothelial cell riding over more effete cells in PPD. $(S E M \times 6,000)$

observations that some ultrastructural features are similar, ${ }^{2}$ and that within one family some patients will only have a few vesicles and be diagnosed as having PPD, whilst others have severe stromal and epithelial oedema, ${ }^{1}$ which may be labelled as CHED. ${ }^{16}$ The majority of posterior polymorphous dystrophy patients with limited posterior corneal involvement, often retain normal vision, and are free from symptoms, ${ }^{1}$ and therefore will not usually come to keratoplasty. However, these ten cases, some of which, ${ }^{17,18,19}$ have been the subject of previous study (although further material has been examined for the current review), all required keratoplasty for decompensation or increasing stromal and epithelial oedema, coupled in some cases, with band keratopathy. The ten cases of PPD tended to present later than our 20 cases of CHED, many of whom, especially the recessives, presented within the first ten years of 


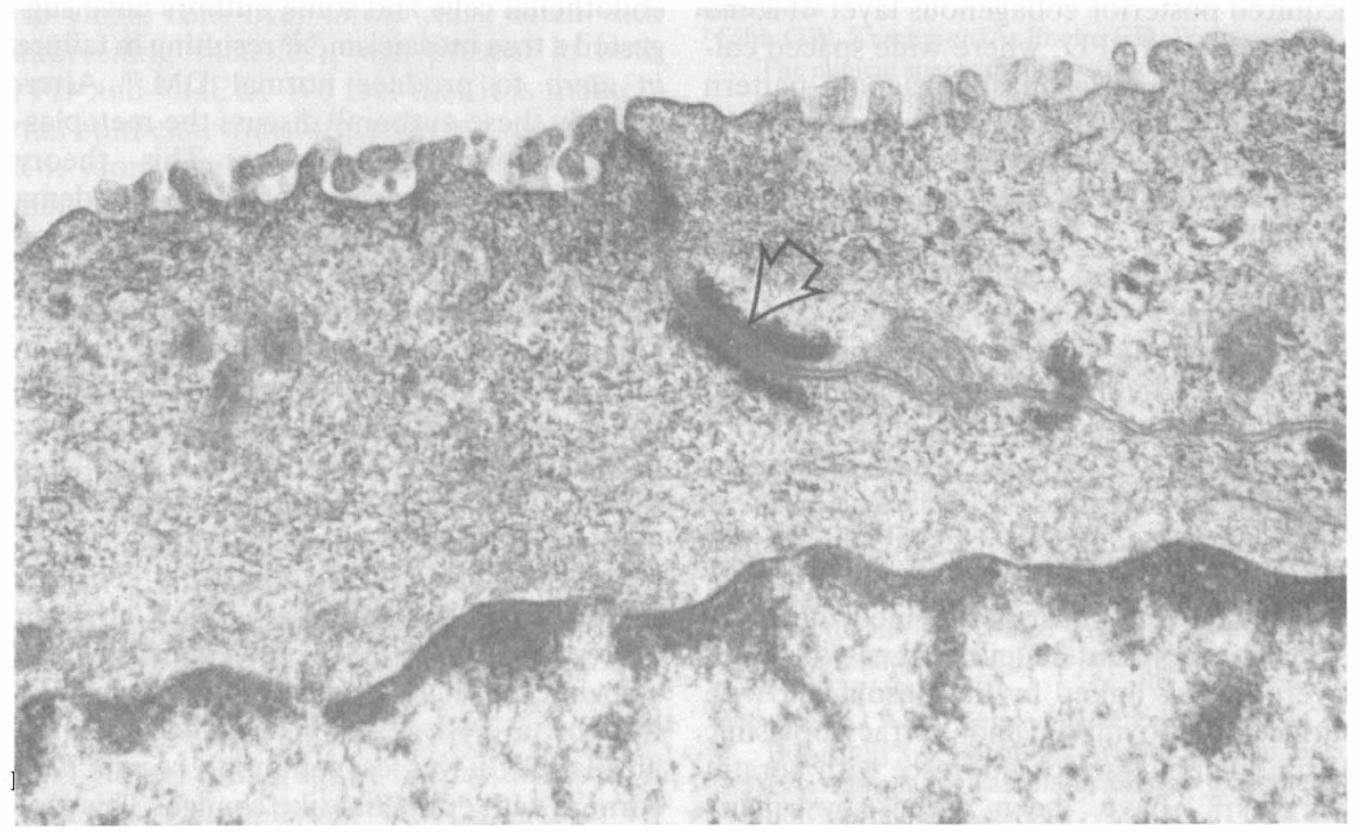

life. Some of the CHED patients were initially misdiagnosed as congenital buphthalmos, and treated inappropriately with drainage surgery, although all had normal intraocular pressure and corneal diameters. Such confusion did not arise in the PPD patients, who had classical signs of PPD, including snail tracks and vesicles, many of whom had been followed clinically for years, before requiring surgery.

This picture of the heterogenous endothelium in PPD and Descemet's membrane, gained clinically using the slit lamp and confirmed with specular microscopy, is amply demonstrated in our study and others, when the nodular or warty ${ }^{18,20}$ appearance and irregularity of Descemet's membrane has also been commented on. ${ }^{6}$

Although primarily an endothelial dystrophy, the changes at the level of Descemet's membrane excite great interest. The development of Descemet's membrane, regulated by the subjacent endothelium, derived from neural crest cells, ${ }^{21}$ normally results in an anterior banded zone (ABZ) formed in utero and a posterior non-banded zone, which in the absence of any pathological change except increasing senescence, is laid down as a fairly homogenous posterior non-banded zone (PNBZ) composed predominantly of basement membrane material with some focal fibrillar or widespaced collagen. ${ }^{13}$ Since the structure of Descemet's membrane and any subsequent posterior thickening may be regarded as a record ${ }^{22}$ of insults or endothelial cell behaviour, the findings of normal $\mathrm{ABZ}$ in our patients with PPD in contrast with the recessive patients with CHED, indicates that in our group of PPD patients endothelial cells dysfunction occurred after the eighth month of development. In contrast to our cases, a case of PPD in a 2.1/2 month old child was reported, ${ }^{23}$ in which no normal Descemet's membrane was discernible. The thinning of the PNBZ in many of the patients with PPD and dominant CHED, indicates that endothelial cell dysfunction can occur early in life in both of these diseases but the non-specific pathological response of secretion of an abnormal posterior collagenous layer of the fibrillary type is also similar in both. The cross linking bridges formed by filaments in the normal ABZ, leading to the characteristic banding, are grotesquely caricatured in the 
acquired posterior collagenous layer of some of our cases of PPD, where wide spaced collagen also forms a quasi-hexagonal pattern layered through basement membrane-like material, mimicking $\mathrm{ABZ}$ in a coarsened fashion. Occasional fibrils of fine collagen are also present.

Similar banded material was shown amongst other conditions. ${ }^{14}$ However, as Waring has recently written, the cornea is only capable of a limited number of responses to different stimuli. ${ }^{24}$ No fibrocellular posterior collagenous layer was seen in any of our cases of PPD and CHED.

If snail tracks and vesicles are the clinical hallmarks of PPD then epithelialisation of endothelial cells must be deemed the pathological equivalent. ${ }^{5}$ Development of multilayered cells, linked by desmosomes, ${ }^{25}$ with surface microvilli6,25 and intracytoplasmic $10 \mathrm{~nm}$ filaments which fluoresce with keratin markers, ${ }^{25}$ have been seen by many authors. ${ }^{5,6,9,19}$ Others have seen attenuated endothelial cells and some authors have suggested a true mosaicism, ${ }^{6,26}$ resulting in failure in utero to produce normal DM. ${ }^{26}$ Alternatively these authors ${ }^{26}$ discuss the metaplastic transformation ${ }^{2}$ theory. This theory suggests that the degree of corneal oedema and hence the clinical diagnosis depends on the failure to evolve epithelial-like cells, which presumably maintain the status quo for longer, as they are more commonly seen in PPD. One of our cases of recessive CHED in a young child did have epithelial-like cells, over a normal focally thinned $\mathrm{ABZ}$ and thin PNBZ, which by the time of his second keratoplasty had been thickened ${ }^{4}$ (case 20 ) by normal posterior NBZ. Epithelial cells can therefore be seen to preserve DM; however others have found that epithelial cells can be associated with abnormal DM and accumulation of a PCL. ${ }^{7,11}$ Non-specific birds-nest fibrillary PCL was less commonly seen in PPD corneas with epithelial-like change. This type of PCL was however seen when multilayering

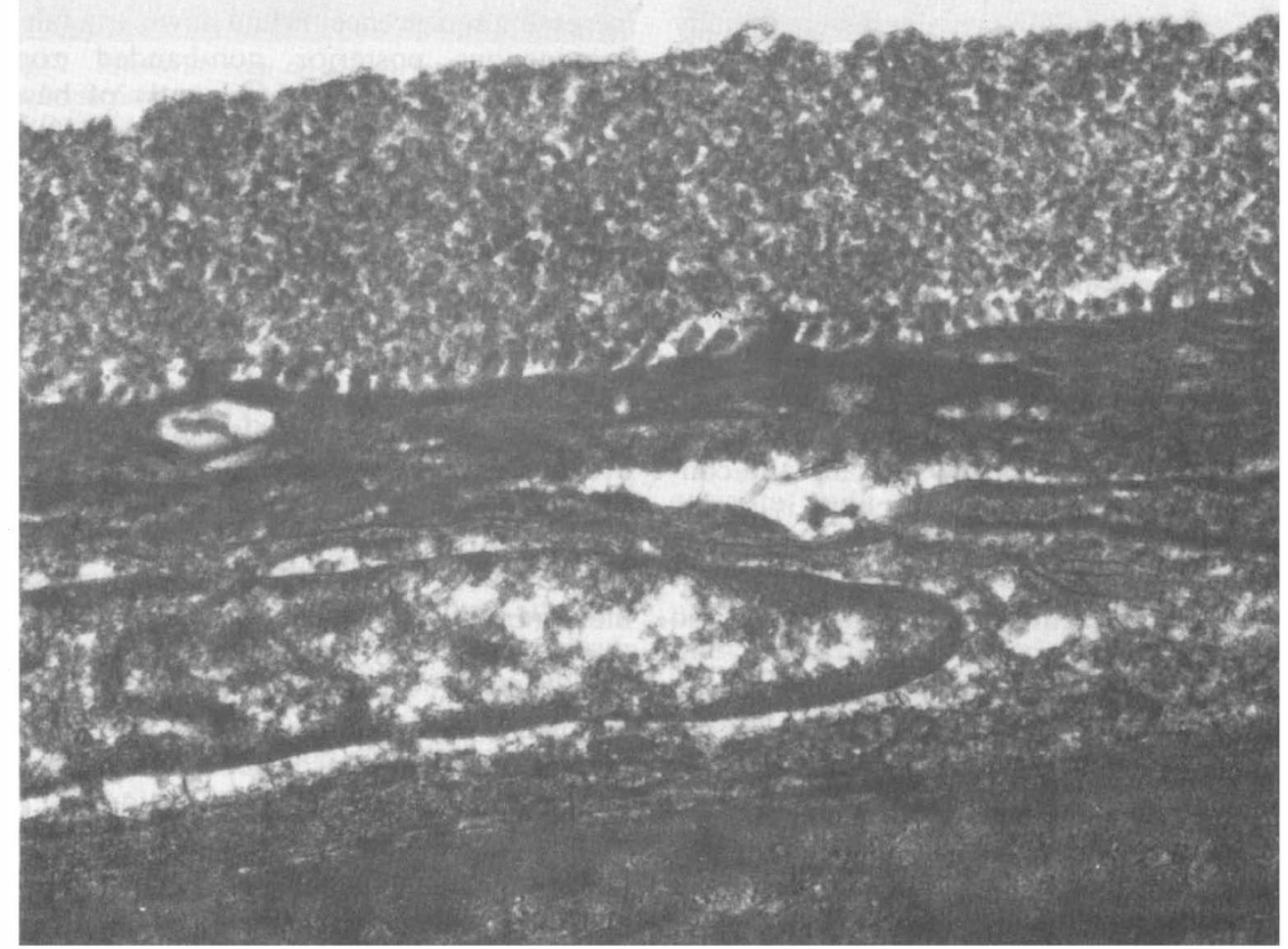

Fig. 5 Viscous layer in PPD. $(T E M \times 12,000)$ 
(of viable cells over effete cells, often with intervening vacuoles), was present in both PPD and CHED. We feel that it is unlikely that epithelial transformation is a mosaicism; it is more likely that although PPD usually manifests itself in later life, in some cases associated with early presentation, the cells are metaplastically transformed earlier. This fits with the non-specific development of epithelial cells in unrelated conditions, ${ }^{27}$ and the ability of endothelial cells in culture to develop desmosomes and intracytoplasmic filaments when growing out into a third dimen$\operatorname{sion}^{28}$ rather than as a monolayer. Development of desmosomes rather than 'zipper-like junctions' or prominent zonulae occludens appears to be a phenomenon arising as a result of multilayering, or vice versa, multilayering depends on the development of greater anchorage points, so that over-riding cells require such junctions in order not to topple off.

This ability of corneal endothelial cells to adapt opportunistically both their morphology and physiology ${ }^{28}$ to altered circumstances may explain the heterogeneity of cell appearance in both PPD and CHED. ${ }^{4}$ If the corneal endothelial cell's response is governed by local and different stimuli, resulting in an apparently similar appearance in several diseases, then some of the current controversy about the apparent similarities between PPD and the ICE syndrome may be resolved.

The role of microvilli in transformed endothelial cells has yet to be determined. They were seen in vast quantities in several of our cases and it is fascinating to see the preservation of the viscous layer in one case of PPD, was apparently due to the mat of microvilli tangling up the postulated glycosaminoglycan layer that is usually removed during processing (Fig. 5). Viscous layer has recently been preserved by special techniques in cats ${ }^{29}$ and has been seen in man by SEM. The thicker layer seen in our case of PPD may be as a result of increased secretion or greater tendency of the viscous layer to persist amongst the forest of microvilli.

We would like to thank Mr. T. A. Casey for permission to include some of his patients.
References

${ }^{1}$ Cibis GW, Krachmer JA, Phelps CD, Weingeist TA: The clinical spectrum of posterior polymorphous dystrophy. Arch Ophthalmol 1977, 95; 1529-37.

${ }^{2}$ Chan C, Green WR, Barraquer J, Barraquer Somers E, de la Cruz ZC: Similarities between posterior polymorphous and congenital hereditary endothelial dystrophy: a study of 14 buttons in 11 cases. Cornea 1982, 1: 155-72.

${ }^{3}$ Bahn CF, Falls HF, Varley GF, Meyer RF, Edelhauser HF, Bourne WM: Classification of corneal endothelial disorders based on neural crest origin. Ophthalmology 1984, 91: 558-63.

${ }^{4}$ Kirkness C, McCartney A, Rice NSC, Garner A, Steele ADMcG: Congenital hereditary corneal oedema of Maumanee, its clinical features, management and pathology. $\mathrm{Br} J$ Ophthalmol 1986, 71: 130-44.

${ }^{5}$ Krachmer JH: Posterior polymorphous corneal dystrophy: a disease characterised by epithelial-like endothelial cells which influence management and prognosis. Tr Am Ophthalmol Soc 1985, 83: 413-72.

${ }^{6}$ Henriques AS, Kenyon KR, Dohlman $\mathrm{CH}$, Boruchoff SA, Forster SL, Meyer RF, Hanninen LA: Morphological characteristics of posterior polymorphous dystrophy. A study of nine corneas and a review of the literature. Surv Ophthalmol 1984, 29: 139-47.

${ }^{7}$ Bourgeois J, Shields B, Thresher R: Open angle glaucoma associated with posterior polymorphous dystrophy. Ophthalmology 1984, 91: 420-3.

${ }^{8}$ Presberg SE, Quigley HA, Forster RK, Green WR: Posterior Polymorphous Corneal Dystrophy. Cornea 1986, 4: $239-48$.

${ }^{9}$ Boruchoff SA and Kuwabara T: Electron microscopy of posterior polymorphous degeneration. Am J Ophthalmol 1971, 72: 879-87.

${ }^{10}$ Cibis GW, Krachmer JH, Phelps CD, Weingeist TA: Irido-corneal adhesions in posterior polymorphous dystrophy. Trans $1 \mathrm{~m}$ Acad Ophthalmol Otolaryngol 1976, 81: 770-7.

11 Grayson M: The nature of hereditary polymorphous dystrophy of the cornea: its association with iris and anterior chamber dysgenesis. Trans Am Ophthalmol Soc 1974, 72: 516-59.

${ }^{12}$ Sabetes R, Krachmer JH, Weingeist TA: Ocular findings in Alport's syndrome. Ophthalmologica (Basel) 1983, 186: 204-10.

${ }^{13}$ Murphy C, Alvarado J, Juster R: Prenatal and postnatal growth of the human Descemet's membrane. Invest Ophthalmol Vis Sci 1984, 25: 1402-15.

${ }^{14}$ Waring GO: Posterior collagenous layer of the cornea. Ultrastructural classification of abnormal tissue posterior to Descemet's membrane in $\mathbf{3 0}$ cases. Arch Ophthalmol 1982, 100: 122-34.

${ }^{15}$ Pouliquen Y, Graf B, Hamada R, Giraud JP, Clay R, Arrata MW, Frouin MA: Dystrophie congénitale de la cornée. Etude en microscopie optique et en microscopie électronique. Arch Ophthalmol (Paris) 1972, 32: 391-414. 
${ }^{16}$ Levenson JE, Chandler JW, Kaufman HE: Affected asymptomatic relatives in congenital hereditary endothelial dystrophy. Am J Ophthalmol 1973, 76: $967-71$.

${ }^{17}$ Liakas GM and Casey TA: Posterior polymorphous keratopathy. Br J Ophthalmol 1978, 62: 39-45.

${ }^{18}$ Morgan G and Patterson A: Pathology of posterior polymorphous degeneration of the cornea. $\mathrm{Br} J$ Ophthalmol 51: 433-7.

${ }^{19}$ Tripathi RC, Casey TA, Wise G: Hereditary posterior polymorphous dystrophy, an ultrastructural and clinical report. Trans Ophthalmol Soc UK 1974, 94: 211-25.

${ }^{20}$ Hogan MJ and Bietti G: Hereditary deep dystrophy of the cornea (polymorphous). Am J Ophthalmol 1969, 68: 777-88.

21 Johnston MC, Noden DM, Hazelton RD, Coulombre JL, Coulombre AJ: Origins of avian ocular and periocular tissues. Exp Eye Res 1979, 29: $27-43$.

22 Waring GO, Bourne WM, Edelhauser HF, Kenyon $\mathrm{K}$ : The corneal endothelium. Normal and pathologic struture and function. Ophthalmology 1982, 89: 531-90.

${ }^{23}$ de Felice GP, Braidotti P, Viale G, Bergamini, F,
Vinciguerra P: Posterior polymorphous dystrophy of the cornea. An ultrastructural study. Graefe's Arch Clin Exp Ophthalmol 1985, 223: 265-71.

${ }^{24}$ Waring GO and Rodrigues MW: Patterns of pathological response in cornea. Surv Ophthalmol 1987, 31: 262-6.

${ }^{25}$ Rodrigues MM, Sun T-T, Krachmer J, Newsome D: Epithelialisation of the corneal endothelium in posterior polymorphous dystrophy. Invest Ophthalmol Vis Sci 1980, 19: 832-5.

${ }^{26}$ Richardson WP and Hettinger ME: Endothelial and epithelial-like cell formations in a case of posterior polymorphous dystrophy. Arch Ophthalmol 1985, 103: 1520-4.

27 Johnson BL and Brown SI: Congenital epithelialisation of the posterior cornea. Am J Ophthalmol 1976, 82: 83-9.

${ }^{28}$ Lowry GM: Corneal endothelium in vitro: characterisation by ultrastructure and histochemistry. Invest Ophthalmol 1966, 5: 355-66.

${ }^{29}$ Carrington SD, Alexander RA, Hiscott P, Grierson I: The viscous layer overlying the corneal posterior epithelium of the domestic cat. J Anat 1987, 153: 77-92. 